\title{
Behavior Therapy for Tic Disorders: an Evidenced-Based Review and New Directions for Treatment Research
}

\author{
Joseph F. McGuire ${ }^{1}$ Emily J. Ricketts ${ }^{1}$. John Piacentini ${ }^{1}$ - Tanya K. Murphy ${ }^{2,3,7}$. \\ Eric A. Storch ${ }^{2,3,4,5,6,7}$ - Adam B. Lewin ${ }^{2,3,5}$
}

Published online: 14 September 2015

(C) Springer International Publishing Switzerland 2015

\begin{abstract}
Behavior therapy is an evidenced-based intervention with moderate-to-large treatment effects in reducing tic symptom severity among individuals with persistent tic disorders (PTDs) and Tourette's disorder (TD). This review describes the behavioral treatment model for tics, delineates components of evidence-based behavior therapy for tics, and reviews the empirical support among randomized controlled trials for individuals with PTDs or TD. Additionally, this review discusses several challenges confronting the behavioral management of tics, highlights emerging solutions for these challenges, and outlines new directions for treatment research.
\end{abstract}

Keywords Tourette's disorder · Persistent tic disorder . Treatment outcome $\cdot$ Comprehensive Behavioral Intervention

This article is part of the Topical Collection on Tourette's Syndrome

Joseph F. McGuire

jfmcguire@mednet.ucla.edu

1 Semel Institute of Neuroscience and Human Behavior, University of California Los Angeles, 760 Westwood Plaza, 48-228B, Los Angeles, CA 90095, USA

2 Department of Pediatrics, University of South Florida, Tampa, FL, USA

3 Departments of Psychiatry and Behavioral Neurosciences, University of South Florida, Tampa, FL, USA

4 Department of Health Policy and Management, University of South Florida, Tampa, FL, USA

5 Department of Psychology, University of South Florida, Tampa, FL, USA

6 Rogers Behavioral Health - Tampa Bay, Tampa, FL, USA

7 All Children's Hospital, Johns Hopkins Medicine, St. Petersburg, FL, USA for Tics $\cdot$ Habit reversal training $\cdot$ Tourette's syndrome · Developmental disorders

\section{Introduction}

Tics are sudden rapid non-rhythmic motor movements or vocalizations that can be simple (rapid, meaningless) or complex (purposeful, orchestrated) in nature [1]. Tics are relatively common among school-aged youth for brief periods of time but often do not continue beyond 6 months [2]. A chronic or persistent tic disorder (PTD) is characterized by the presence of either a single or multiple motor or vocal tic(s), but not both, that persisted longer than a year, with a diagnosis of Tourette's disorder being conferred when both motor and phonic tics are present (although not necessarily concurrently) for longer than a year [1]. Persistent tic disorders and Tourette's disorder (collectively referred to as PTDs henceforth) affect approximately $0.4-1.6 \%$ of youth $[3,4]$. For youth with PTD, symptoms typically onset around 6 years of age [5] and exhibit a fluctuating course with peaks in symptom severity that stabilize over a period of weeks [6]. For the majority of youth, tics reach their greatest severity in adolescence-increasing in number, type, and frequency - but subside in early adulthood in many cases $[5,7]$. Tic symptoms show minimal difference between youth and adults with PTD [8], with the most common bothersome tics including eye blinking, head jerks, sniffing, throat clearing, and other complex motor tics [9•]. In addition to tics, individuals with PTD typically present with co-occurring psychiatric disorders [e.g., anxiety disorders, attention deficit-hyperactivity disorder (ADHD), obsessivecompulsive disorder (OCD)] [10-13], mood and behavioral problems (e.g., disruptive behaviors, rage attacks, anger problems, suicidal thoughts, and/or behaviors) [14-16], and social difficulties (e.g., peer victimization, social deficits, low self- 
concept) [17-22] (also, see Hanks et al. [22] in this issue). Tics and co-occurring problems can cause individuals with PTD to experience significant impairment [21, 23, 24] and a poor quality of life $[25,26]$. Thus, effective treatments are needed for individuals with PTD to efficiently manage their tics and co-occurring symptoms.

Historically, tic symptom severity has been managed using psychotropic medications, such as antipsychotic agents and/or alpha-2 agonists [27]. A meta-analysis of five randomized controlled trials (RCTs) of antipsychotic medications identified a significant but moderate reduction in tic severity relative to placebo [effect size $=0.58$ ], with no significant difference between medication types [28]. Additionally, a meta-analysis of six RCTs of alpha-2 agonist medications identified a statistically significant albeit small reduction in tic severity relative to placebo (effect size $=0.31$ ) that was moderate when limited to RCTs in which individuals had both PTD and ADHD (effect size $=0.68$ ) [28]. Despite their efficacy, these medications are often accompanied by side effects that can limit long-term use [27]. Moreover, while medication management significantly reduces tic severity, some troublesome tics may remain. Thus, individuals with PTD have to confront and cope with tics even when receiving evidence-based pharmacotherapy.

In addition to pharmacotherapy, behavior therapy has demonstrated success in reducing tic severity for individuals with PTD in RCTs [29, 30, 31••, 32-36] and can serve as a standalone intervention or augmentation strategy to existing pharmacotherapy. Indeed, professional organizations have recommended behavior therapy as the first-line intervention for youth with PTD who have mild-to-moderate tic severity [37-39]. This paper provides an evidence-based review of behavior therapy to manage tic symptoms for individuals with PTDs. This paper also discusses the challenges confronting the behavioral management of tics, highlights emerging solutions for these challenges, and outlines new directions for treatment research.

\section{Behavior Therapy}

Behavioral interventions for tics have existed for several decades, with early case reports having been published in the 1960s and 1970s [40, 41]. Although initial case reports suggested tics to be nervous habits [40], more recent conceptualizations of behavioral interventions acknowledge the neurobiological basis of the condition [42, 43]. Multiple types of behavioral interventions have been evaluated in RCTs for the treatment of individuals with PTD [e.g., habit reversal training (HRT), mass negative practice (MNP), awareness training (AT), exposure response prevention (ERP)] [38], but only HRT and its successor the Comprehensive Behavioral Interventions for Tics (CBIT) have consistently demonstrated efficacy in RCTs and meta-analyses [44•].
HRT is a multiple component intervention that can include psychoeducation, AT, competing response training, generalization training, self-monitoring, relaxation training, behavioral rewards, motivational procedures, and social support [45]. The therapeutic components of HRT are detailed in Table 1. Although it can include multiple components, the core therapeutic skills of HRT are AT, competing response training, and social support [46]. The CBIT utilizes these core HRT components along with relaxation training and behavioral rewards and incorporates functional assessments and function-based interventions to mitigate daily life factors that can exacerbate tic symptoms [43]. Table 1 describes the functional assessment and interventions in greater detail.

Behavioral Treatment Model Behavioral therapies like HRT and CBIT are based on the belief that tics have a neurobiological basis but that both external and internal cues can serve as antecedents that influence tic symptoms [43]. External cues can include specific activities (e.g., playing sports, musical instruments, doing paperwork) and environmental/situational situations (e.g., returning home, specific family members), with internal cues commonly including premonitory urges and/or internal mood states (e.g., anxiety). Individuals with PTD experience corresponding consequences associated with external and internal factors that can subsequently reinforce tic symptoms. For example, a premonitory urge is an internal sensation that individuals describe as an aversive "urge," "feeling," "impulse," and/or "pressure" that often precedes a tic $[47,48]$. Individuals with PTD report that the performance of the tic and/or multiple tics alleviates the distressing premonitory urge [47]. Consequently, the tic becomes negatively reinforced because it produces a reduction in the aversive sensation of the premonitory urge. In HRT and CBIT, individuals learn to identify antecedents to tic symptoms and implement competing responses that break the negative reinforcement cycle. This allows for individuals to habituate to internal aversive triggers like premonitory urges and modification of consequences for external factors (e.g., avoidance of homework or social situations due to increased tics).

Empirical Evidence Multiple case reports have highlighted the benefit of behavior therapy for individuals as young as 5 [49] and old as 75 years of age [50]. Eight published RCTs have evaluated the efficacy of behavior therapy relative to either waitlist conditions [30,34] or an active comparison condition in youth and adults [e.g., MNP, ERP, psychoeducation and supportive therapy (PST)] [29, 31••, 32, 33, 35, 36]. Azrin and colleagues (1980) compared HRT and MNP using a one- to two-session treatment protocol in 22 youth and adults [29]. MNP involves the voluntary rapid and repeated performance of tics for a defined period of time that is interspersed with brief periods of rest [38]. Azrin and colleagues found that participants receiving HRT exhibited a 


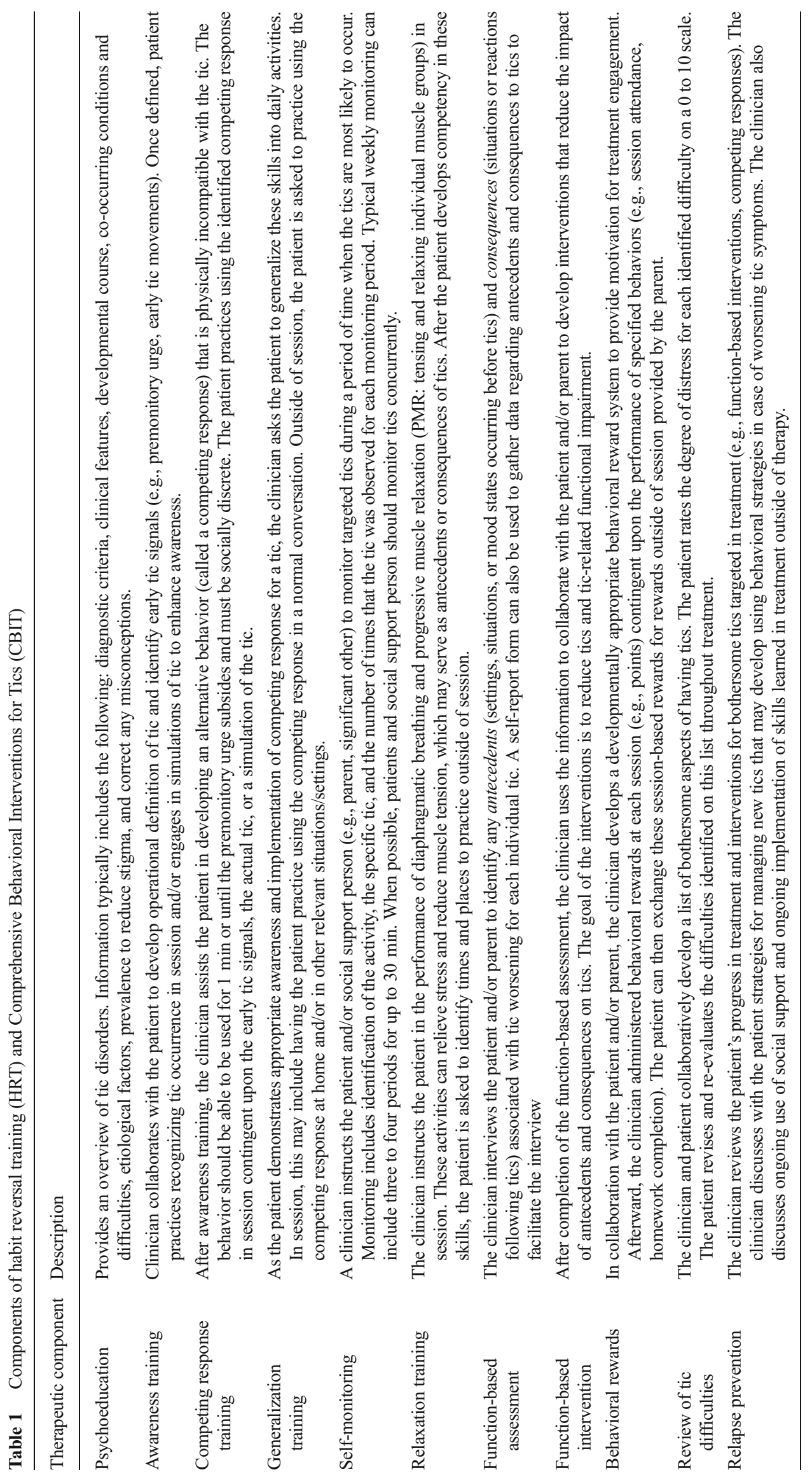


$92 \%$ reduction in tic frequency relative to a $33 \%$ reduction in tic frequency among the MNP condition 4 weeks posttreatment [29].

Azrin and Peterson (1990) compared HRT to a waitlist condition in ten youth and adults with PTD, with participants in the HRT condition receiving an average of seven sessions over a 4-month period [30]. Using tic frequency counts directly observed within the clinic, Azrin and Peterson found that tic frequency reduced by an average of $93 \%$ in the HRT condition compared to an average $14 \%$ reduction waitlist group [30]. O'Connor and colleagues (2001) compared a 13-session HRT treatment package to a waitlist control condition among 69 individuals with PTD [34]. O'Connor and colleagues observed that participants receiving HRT exhibited greater reductions in video observations of tic frequency and severity compared to the waitlist control condition [34].

In 2003, Wilhelm and colleagues compared the efficacy of HRT relative to PST in a 14-session treatment protocol for 32 adults with PTD [35]. Wilhelm and colleagues found a large within-group effect size for reduction of tic severity in the HRT group $(d=1.50)$, with no meaningful within-group effect observed in the PST group $(d=-0.03)$ [35]. Verdellen and colleagues (2004) compared 10 sessions of HRT to 12 sessions of ERP in 43 youth and adults with PTD [33]. ERP is a behavioral intervention similar to HRT and CBIT but differs in its approach to managing tic symptoms. In ERP, participants are exposed to sensations (e.g., premonitory urges) and stimuli that elicit tics for a prolonged period of time and practice suppressing/resisting the tic (rather than engaging in a competing response) $[33,38]$. Through repeated exposure and resistance, individuals are believed to habituate to the sensations and stimuli that elicit the tics. Similar to HRT and CBIT, this is believed to discontinue the negative reinforcement cycle wherein the individual experiences relief in aversive sensation (i.e., the premonitory urge) following the tic. The ERP group exhibited an averaged 8.6-point reduction on the Yale Global Tic Severity Scale (YGTSS) compared to an average 4.4 point reduction on the YGTSS in the HRT group. Although these group differences approached statistical significance ( $p=0.06$ ), the noted difference in therapeutic contact between treatment conditions confounds these findings (12 120-min ERP sessions versus 10 60-min HRT sessions) [33]. In 2006, Deckersbach and colleagues compared a 12session treatment protocol of HRT to PST in 30 adults with PTD [36]. Although no significant difference in tic severity was observed at pretreatment, Deckersbach and colleagues found that participants in the HRT condition had lower tic severity at the mid-treatment and post-treatment assessments compared to the PST condition [36].

Piacentini and colleagues (2010) conducted a large-scale multicenter RCT of eight sessions of CBIT compared to eight sessions of PST over 10 weeks in 126 youth with PTD [31••]. Piacentini and colleagues found that CBIT was associated with a 7.6-point decrease on the YGTSS compared to a 3.5 -point decrease in the PST condition on the YGTSS $(p<0.001$, effect size $=0.68)[31 \bullet \cdot$. On the Clinical Global Impression of Improvement (CGI-I) [51], $52.5 \%$ of participants in the CBIT condition and $18.5 \%$ of participants in the PST condition exhibited a positive response to treatment $[31 \bullet \cdot]$. Therapeutic gains observed during the acute treatment period were found to maintain after 6 months, with no adverse consequences [31••, 52]. In 2012, Wilhelm and colleagues (2012) conducted a parallel large-scale multicenter RCT of eight sessions of CBIT compared to eight sessions of PST over 10 weeks in 122 older adolescents and adults with PTD [32]. Wilhelm and colleagues found that participants in the CBIT group had a $25.8 \%$ decrease on the YGTSS compared to an $11.5 \%$ decrease in the PST group $(p<0.001$, effect size $=0.57$ ) [32]. On the CGI-I, $38.1 \%$ of participants in the CBIT condition and $6.8 \%$ in the PST condition exhibited a positive response to treatment [32]. Treatment gains were observed up to 6 months after acute treatment [32]. Follow-up analyses across these two large CBIT trials identified that CBIT was efficacious across all identified tic symptom clusters [8]. An individualized examination of treatment response of specific bothersome tics and tic characteristics in these CBIT trials found that specific tics were more likely to improve and remit with CBIT relative to PST, with broader tic characteristic analyses revealing that tics with a premonitory urge exhibited greater improvement and remission with CBIT compared to PST [9•].

In 2014, McGuire and colleagues conducted a random effects meta-analysis of behavior therapy RCTs and examined moderators of treatment effects [44•]. Behavior therapy had a moderate-to-large effect (effect size $=0.67-0.94$ ) relative to comparison conditions [44•]. Additionally, participants receiving behavior therapy were more likely to exhibit a treatment response relative to comparison conditions (odds ratio $=$ 5.77), with a number needed to treat of three [44•]. Moderator analysis identified that greater mean participant age and average number of therapy sessions were associated with larger treatment effects, whereas an increased percentage of participants with co-occurring ADHD was associated with smaller treatment effects $[44 \cdot]$.

\section{Challenges Confronting Behavior Therapy for Tics}

Although behavior therapy has considerable empirical support for reducing tic symptom severity, several challenges exist to its widespread use and implementation as a first-line intervention for individuals with PTD. This section outlines these challenges and highlights potential emerging solutions. 
Clinician Concerns Regarding Possible Negative Consequences of Behavior Therapy Some clinicians have expressed concerns that behavior therapy may have unintended negative consequences for individuals with PTD. These include concerns that focusing attention on tics will make them worse, that tic suppression produces "rebound" effects, and that behavior therapy may result in symptom substitution [53]. Although these concerns are well intentioned, there exists empirical evidence that contradicts many of these misconceptions. First, there is a belief among health care providers that either talking about tics [54] or focusing attention on tics will make them worse [55]. While tic-related discussions and direct attention to tics can momentarily increase tic frequency $[56,57]$, AT that includes focused attention on individual tics and self-monitoring of tics has been shown to actually produce modest reductions in tics [46]. Second, a considerable percentage of health care providers believes that tic suppression strategies may inadvertently produce increased tic frequency above initial levels referred to as a "rebound effect" [54]. The best evidence to empirically evaluate this concern comes from Verdellen and colleagues (2007) who compared tic frequency in a 15-min video observation before and after ERP therapy sessions in which participants practiced tic suppression [58]. After each one of the therapy sessions, the mean post-session tic counts were lower than baseline tic frequency, demonstrating that tic suppression activities did not result in worsening of tic symptoms [58]. Third, some clinicians have raised concerns that behavior therapy can result in symptom substitution based on anecdotal case reports [54, 59, 60]. Symptom substitution is a long-standing concern that dichotomizes psychodynamic and behavioral therapies [61]. Symptom substitution refers to the belief that the behavioral treatment of one tic may result in the appearance of a new tic and/or an increase in severity of other non-targeted tics. For example, if an arm tic was treated with behavior therapy, the concern may be that the patient may develop a new tic (e.g., leg kick) in response to suppressing their original tic and/or experience a worsening in severity of non-targeted tics. Despite possible evidence in among case reports [59, 60], systematic empirical evaluations have found no evidence of symptom substitution in response to behavior therapy for tics for individuals with PTD [62].

Limited Accessibility and Availability While behavior therapy has demonstrated its efficacy for managing tic severity in structured RCTs, there are a limited number of clinicians trained in evidence-based behavioral practice [63]. This poses a considerable challenge for managing tics with behavior therapy in community settings, as there is likely availability and access to trained treatment providers $[63,64]$. As a result, many individuals with PTD may be prohibited from either receiving behavior therapy and/or experience long-wait periods prior to treatment initiation. Several innovative solutions have been explored to address this challenge. First, the Tourette Association of America (previously the Tourette Syndrome Association) and the US Centers for Disease Control and Prevention have collaborated to develop training opportunities to increase the number of clinician's trained in evidence-based behavior therapy [64]. Second, several studies have examined novel treatment delivery modalities to increase access and availability of behavior therapy. These innovations include intensive behavior therapy treatment protocols [65, 66] and telemedicine approaches to delivering behavior therapy [67-70]. Third, given the limited number of mental health professionals trained in behavior therapy, other professionals such as physical therapists have been exploring the possibility of conducting behavior therapy for PTD. Rowe, Yuen, and Due (2013) conducted an open-label trial of eight sessions of CBIT for PTD conducted by physical therapists in 30 youth with PTD [71]. Rowe and colleagues found that youth exhibited a reduced number of tics and had improved scores on the Parent Tic Questionnaire [71, 72]. Although these collective approaches have shown considerable promise, future research is needed to continue to explore approaches to increasing the availability and accessibility of behavior therapy for individuals with PTD.

\section{Limited Therapeutic Response to Behavior Therapy} Although many individuals receiving behavior therapy experience significant reductions in tic severity, these behavioral interventions do not often result in complete tic remission. Indeed, a positive response to treatment among individuals with PTD corresponds with a $25-35 \%$ reduction in tic severity on the YGTSS $[73,74]$. Thus, there is a clear need to enhance therapeutic outcomes with behavioral interventions. Several possible approaches to enhance therapeutic outcomes may prove useful for individuals with PTD based on the limited available evidence. First, the addition of adjunctive therapeutic components to existing evidence-based behavioral interventions may prove beneficial. Franklin and colleagues (2011) [75] found that adding acceptance and commitment therapy (ACT) to evidence-based behavior therapy yielded additional benefit relative to evidence-based behavior therapy alone in a small pilot study of youth with PTD [75]. Second, the augmentation of behavioral interventions with pharmacotherapy may result in enhanced therapeutic outcomes. Lyon and colleagues (2010) examined whether methylphenidate (MPH) enhanced youth's ability to suppress tics relative to placebo in a RCT [76]. Although Lyon found minimal difference in youth's ability to enhance tic suppression between groups [76], it is important to note that tic suppression is distinctly different from evidence-based behavior therapies. Thus, the combination of MPH and behavior therapy may still prove useful, as co-occurring ADHD was found to attenuate treatment effects in a recent meta-analysis [44•]. Finally, the use of cognitive enhancers may serve as another possibility to 
augment therapeutic outcomes with behavior therapy. Broadly, cognitive enhancers are compounds that are believed to augment psychosocial interventions to produce expedited and/or enhanced therapeutic benefit (e.g., d-cycloserine). Cognitive enhancers have demonstrated some promise among related disorders like OCD in enhancing therapeutic outcome and expediting treatment gains [77]. Despite the possible potential of all three augmentation approaches, considerable future research is needed to investigate evidence-based augmentation strategies for behavior therapy.

\section{Limited Treatments Targeting Other Problems Encoun-} tered by Individuals with PTD The predominant focus of pharmacological and behavioral interventions for individuals with PTD has been reduction in tic symptom severity. This treatment approach is predicated on the assumption that tic severity is predominantly responsible for the distress, impairment, and poor quality of life experienced by individuals with PTD. While many individuals experience significant improvement with pharmacological or behavioral interventions, a considerable percentage of individuals continues to experience distress and impairment from tics and associated problems (e.g., social problems, social deficits, peer victimization, poor self-perception). Moreover, as complete symptom remission is infrequent with either intervention, individuals have to learn effective coping skills to manage daily challenges associated with tics. Taken together, there is a clear need for evidence-based interventions to reduce impairment, improve the quality of life, and develop effective coping strategies among individuals with PTD. Although recognized as an important aspect of treatment in evidencebased practice parameters [37], there have only been two studies that have evaluated treatments to address these concerns. First, Storch and colleagues (2012) developed a modular cognitive behavioral therapy (CBT) called "living with tics" (LWT) that aimed to promote resiliency and coping skills to manage the psychosocial consequences of tics among youth with PTD [78]. The LWT intervention was evaluated in an open-label case series of eight youth with PTD. Youth exhibited significant reductions in tic-related impairment and tic severity on the YGTSS, as well as experienced improved self-concept and quality of life [78]. On the CGI-I, six of the eight youth $(75 \%)$ were considered treatment responders. Second, McGuire and colleagues (2014) extended the initial findings by Storch and colleagues (2012) by incorporating additional modules into the LWT intervention and evaluated its efficacy compared to a waitlist condition in a RCT of 24 youth with PTD [78, 79]. Youth in the LWT $(n=12)$ group exhibited significantly reduced tic-related impairment on the YGTSS and improved quality of life relative to the waitlist condition [79]. Ten youth (83\%) in the LWT group were considered treatment responders on the CGI-I compared to four youth in the waitlist condition (33\%) [79]. Treatment gains in the LWT group were maintained at a 1-month follow-up assessment [79]. While the modular LWT intervention has demonstrated benefit as a stand-alone treatment, it can also serve as important therapeutic complements to either behavior therapy or pharmacological interventions. Although demonstrating considerable promise, additional research is needed to develop and evaluate further interventions focused on reducing impairment and promoting quality of life among individuals with PTD-especially among adults.

\section{Conclusion}

In summary, behavior therapy is an established evidencebased intervention for the management of tics characteristic of PTD. Behavior therapy has demonstrated its efficacy across multiple RCTs and produces treatment effects comparable to those observed with antipsychotic medications. Indeed, professional organizations recommend behavior therapy as a first-line intervention for individuals with mild-to-moderate tic severity [37], with recent research highlighting the counterintuitive benefit of behavior therapy for tics with premonitory urges that tend to have greater severity [9॰]. While behavior therapy offers the promise of significant tic reduction in the majority of cases, some challenges remain that confront the regular utilization of behavior therapy as a first-line intervention for individuals with PTD. Additionally, further efforts are needed to enhance therapeutic outcomes for individuals who fail to exhibit a clinically meaningful response to behavior therapy and refine related interventions to promote coping skills to manage the adverse psychosocial consequences of tics. Taken together, these challenges highlight the importance of ongoing behavioral treatment research to improve the therapeutic outcomes and quality of life for individuals with PTD.

Acknowledgments This research was supported in part by a grant from the National Institute of Mental Health (NIMH) supporting Dr. Ricketts (T32MH073517). The views expressed within this article represent those of the authors, were not influenced by this funding source, and are not intended to represent the position of NIMH.

\section{Compliance with Ethics Guidelines}

Conflict of Interest Joseph F. McGuire declares he has no conflicts of interest.

Emily J. Ricketts declares she has no conflict of interest.

John Piacentini reports grants from the National Institute of Mental Health, Pfizer, The Pettit Family Foundation; grants and personal fees from Tourette Syndrome Association; and personal fees from Oxford 
University Press, Guilford Publications, International OCD Foundation, and Trichotillomania Learning Center.

Tanya K. Murphy reports grants from Florida Agency for Healthcare Administration, AstraZeneca Research \& Development, Center for Disease Control and Prevention, F. Hoffmann-La Roche Ltd., International OCD Foundation, National Institutes of Health/National Institute of Mental Health, Otsuka Pharmaceuticals, Pfizer, Inc, Psyadon Pharmaceuticals, Inc, Shire Pharmaceuticals, Auspex Pharmaceuticals, and other from Tourette Syndrome Association, outside the submitted work.

Eric A. Storch reports grants from the National Institutes of Health, the Center for Disease Control and Prevention, The Agency for Healthcare Research and Quality, Janssen Scientific Affairs, and All Children's Hospital Research Foundation; grants and other from International OCD Foundation; and other from Springer publishing, American Psychological Association, Lawrence Erlbaum, WileyBlackwell, Rogers Memorial Hospital, Prophase, Inc, and All Children's Hospital Guild Endowed Chair.

Adam B. Lewin reports grants from International OCD Foundation and All Children's Hospital and other from Springer Publishing, Tourette Syndrome Association, Children's Tumor Foundation, Rogers Memorial Hospital, National Institute of Mental Health, the Society for Clinical Child and Adolescent Psychology, University of Central Oklahoma, and Prophase LLC.

Human and Animal Rights and Informed Consent This article does not contain any studies with human or animal subjects performed by any of the authors.

\section{References}

Papers of particular interest, published recently, have been highlighted as:

- Of importance

•- Of major importance

1. American Psychiatric Association. Diagnostic and statistic manual of mental disorders. 5th ed. Arlington: American Psychiatric Publishing; 2013.

2. Cubo E, Gabriel Y, Galan JM, Villaverde VA et al. Prevalence of tics in schoolchildren in central Spain: a population-based study. Pediatr Neurol. 2011;45(2):100-8.

3. Scahill L, Specht M, Page C. The prevalence of tic disorders and clinical characteristics in children. J Obsessive Compuls Relat Disord. 2014;3(4):394-400.

4. Knight T, Steeves T, Day L et al. Prevalence of tic disorders: a systematic review and meta-analysis. Pediatr Neurol. 2012;47(2): 77-90.

5. Bloch MH, Leckman JF. Clinical course of Tourette syndrome. J Psychosom Res. 2009;67(6):497-501.

6. Lin H, Yeh CB, Peterson BS et al. Assessment of symptom exacerbations in a longitudinal study of children with Tourette's syndrome or obsessive-compulsive disorder. J Am Acad Child Adolesc Psychiatry. 2002;41(9):1070-7.

7. Bloch MH, Peterson BS, Scahill L et al. Adulthood outcome of tic and obsessive-compulsive symptom severity in children with Tourette syndrome. Arch Pediatr Adolesc Med. 2006;160(1):65-9.

8. McGuire JF, Nyirabahizi E, Kircanski K et al. A cluster analysis of tic symptoms in children and adults with Tourette syndrome: clinical correlates and treatment outcome. Psychiatry Res. 2013;210(3): 1198-204.
9. McGuire JF, Piacentini J, Scahill L et al. Bothersome tics in patients with chronic tic disorders: characteristics and individualized treatment response to behavior therapy. Behav Res Ther. 2015;70:5663. This study identified the individual response of specific tics and general tic characteristics to behavior therapy. Findings identified specific tics and characteristics associated with a significant reduction in severity to behavior therapy.

10. Specht MW, Woods DW, Piacentini J et al. Clinical characteristics of children and adolescents with a primary tic disorder. J Dev Phys Disabil. 2011;23(1):15-31.

11. Lebowitz ER, Motlagh MG, Katsovich L et al. Tourette syndrome in youth with and without obsessive compulsive disorder and attention deficit hyperactivity disorder. Eur Child Adolesc Psychiatry. 2012;21(8):451-7.

12. Lewin $\mathrm{AB}$, Chang $\mathrm{S}$, Mccracken $\mathrm{J}$ et al. Comparison of clinical features among youth with tic disorders, obsessive-compulsive disorder (OCD), and both conditions. Psychiatry Res. 2010;178(2): 317-22.

13. Freeman RD, Fast DK, Burd L et al. An international perspective on Tourette syndrome: selected findings from 3,500 individuals in 22 countries. Dev Med Child Neurol. 2000;42:436-47.

14. Kraft JT, Dalsgaard S, Obel C et al. Prevalence and clinical correlates of tic disorders in a community sample of school-age children. Eur Child Adolesc Psychiatry. 2012;21(1):5-13.

15. Sukhodolsky DG, Scahill L, Zhang H et al. Disruptive behavior in children with Tourette's syndrome: association with ADHD comorbidity, tic severity, and functional impairment. J Am Acad Child Adolesc Psychiatry. 2003;42(1):98-105.

16. Storch EA, Hanks CE, Mink JW et al. Suicidal thoughts and behaviors in children and adolescents with chronic tic disorders. Depress Anxiety. 2015.

17. McGuire JF, Hanks C, Lewin AB et al. Social deficits in children with chronic tic disorders: phenomenology, clinical correlates and quality of life. Compr Psychiatry. 2013;54(7):1023-31.

18. Storch EA, Murphy TK, Chase RM et al. Peer victimization in youth with Tourette's syndrome and chronic tic disorder: relations with tic severity and internalizing symptoms. J Psychopathol Behav Assess. 2007;29(4):211-9.

19. Zinner SH, Conelea CA, Glew GM et al. Peer victimization in youth with Tourette syndrome and other chronic tic disorders. Child Psychiatry Hum Dev. 2012;43(1):124-36.

20. Hanks CE, McGuire JF, Lewin AB et al. Clinical correlates and mediators of self-concept in youth with chronic tic disorders. Child Psychiatry Hum Dev. 2015;1-11.

21. Conelea CA, Woods DW, Zinner SH et al. The impact of Tourette syndrome in adults: results from the Tourette syndrome impact survey. Community Ment Health J. 2013;49(1):110-20.

22. Hanks CE, Lewin AB, Mutch PJ et al. Social deficits and autism spectrum disorders in Tourette's syndrome. Curr Dev Disord Rep. 2015;1-8.

23. Conelea CA, Woods DW, Zinner SH et al. Exploring the impact of chronic tic disorders on youth: results from the Tourette syndrome impact survey. Child Psychiatry Hum Dev. 2011;42(2):219-42.

24. Storch EA, Lack CW, Simons LE et al. A measure of functional impairment in youth with Tourette's syndrome. J Pediatr Psychol. 2007;32(8):950-9.

25. Storch EA, Merlo LJ, Lack C et al. Quality of life in youth with Tourette's syndrome and chronic tic disorder. J Clin Child Adolesc Psychol. 2007;36(2):217-27.

26. Jalenques I, Galland F, Malet L et al. Quality of life in adults with Gilles de la Tourette syndrome. BMC Psychiatry. 2012;12(1):109.

27. Scahill L, Erenberg G, Berlin CM et al. Contemporary assessment and pharmacotherapy of Tourette syndrome. NeuroRx. 2006;3(2): 192-206.

28. Weisman H, Qureshi IA, Leckman JF et al. Systematic review: pharmacological treatment of tic disorders-efficacy of 
antipsychotic and alpha-2 adrenergic agonist agents. Neurosci Biobehav Rev. 2013;37(6):1162-71.

29. Azrin NH, Nunn RG, Frantz SE. Habit reversal vs negative practice treatment of nervous tics. Behav Ther. 1980;11(2):169-78.

30. Azrin NH, Peterson AL. Treatment of Tourette syndrome by habit reversal: a waiting-list control group comparison. Behav Ther. 1990;21(3):305-18.

31.• Piacentini J, Woods DW, Scahill L et al. Behavior therapy for children with Tourette disorder: a randomized controlled trial. J Am Med Assoc. 2010;303(19):1929-37. A multi-center randomized controlled trial of the Comprehensive Behavioral Intervention for Tics for children and adolescents with Tourette Disorder and/or Chronic Tic Disorders.

32. Wilhelm S, Peterson AL, Piacentini J et al. Randomized trial of behavior therapy for adults with Tourette syndrome. Arch Gen Psychiatry. 2012;69(8):795-803.

33. Verdellen CW, Keijsers GP, Cath DC et al. Exposure with response prevention versus habit reversal in Tourettes's syndrome: a controlled study. Behav Res Ther. 2004;42(5):501-11.

34. O'Connor KP, Brault M, Robillard S et al. Evaluation of a cognitive-behavioural program for the management of chronic tic and habit disorders. Behav Res Ther. 2001;39(6):667-81.

35. Wilhelm S, Deckersbach T, Coffey BJ et al. Habit reversal versus supportive psychotherapy for Tourette's disorder: a randomized controlled trial. Am J Psychiatry. 2003;160(6):1175-7.

36. Deckersbach T, Rauch S, Buhlmann U et al. Habit reversal versus supportive psychotherapy in Tourette's disorder: a randomized controlled trial and predictors of treatment response. Behav Res Ther. 2006;44(8):1079-90.

37. Murphy TK, Lewin AB, Storch EA et al. Practice parameter for the assessment and treatment of children and adolescents with tic disorders. J Am Acad Child Adolesc Psychiatry. 2013;52(12):134159.

38. Verdellen C, Van De Griendt J, Hartmann A et al. European clinical guidelines for Tourette syndrome and other tic disorders. Part III: behavioural and psychosocial interventions. Eur Child Adolesc Psychiatry. 2011;20(4):197-207.

39. Steeves T, McKinlay BD, Gorman D et al. Canadian guidelines for the evidence-based treatment of tic disorders: behavioural therapy, deep brain stimulation, and transcranial magnetic stimulation. Can J Psychiatry / La Rev Can Psychiatr. 2012;57(3):144-51.

40. Clark DF. Behaviour therapy of Gilles de la Tourette's syndrome. Br J Psychiatry. 1966;112(489):771-8.

41. Azrin NH, Nunn RG. Habit-reversal: a method of eliminating nervous habits and tics. Behav Res Ther. 1973;11(4):619-28.

42. Felling RJ, Singer HS. Neurobiology of Tourette syndrome: current status and need for further investigation. J Neurosci. 2011;31(35): 12387-95.

43. Woods DW, Piacentini J, Chang SW et al. Managing Tourette syndrome: a behavioral intervention for children and adolescents. Treatments that work. New York: Oxford University Press; 2008.

44. McGuire JF, Piacentini J, Brennan EA et al. A meta-analysis of behavior therapy for Tourette syndrome. J Psychiatr Res. 2014;50: 106-12. A meta-analysis of randomized controlled trials of behavior therapy for individuals with Tourette Disorder and/or Chronic Tic Disorder. Findings identified a moderate-to-large treatment effect, with comorbid ADHD identified as a moderator associated with attenuated treatment effects.

45. Peterson AL. Psychosocial management of tics and intentional repetitive behaviors associated with Tourette syndrome. In: Woods DW, Piacentini JC, Walkup JT, editors. Treating Tourette syndrome and tic disorders: a guide for practitioners. New York: Guilford Press; 2007. p. 154-84.

46. Woods DW, Miltenberger RG, Lumley VA. Sequential application of major habit-reversal components to treat motor tics in children. J Appl Behav Anal. 1996;29(4):483-93.
47. Leckman JF, Walker DE, Cohen DJ. Premonitory urges in Tourette's syndrome. Am J Psychiatry. 1993.

48. Woods DW, Piacentini J, Himle MB et al. Premonitory urge for tics scale (PUTS): initial psychometric results and examination of the premonitory urge phenomenon in youths with tic disorders. J Dev Behav Pediatr. 2005;26(6):397-403.

49. Prabhakar D. Behavioral management of Tourette's disorder in a 5year-old child. Prim Care Companion for CNS Disord. 2014;16(1).

50. McGuire JF, Storch EA. Behavior therapy for a 75-year-old woman with chronic motor tic disorder. Neuropsychiatry. 2013;3(5):47781.

51. Guy W. Clinical global impressions. In: ECDEU assessment manual for psychopharmacology. Rockville: National Institute for Mental Health; 1976. p. 218-22.

52. Woods DW, Piacentini JC, Scahill L et al. Behavior therapy for tics in children: acute and long-term effects on psychiatric and psychosocial functioning. J Child Neurol. 2011;26(7):858-65.

53. Woods DW, Conelea CA, Walther MR. Barriers to dissemination: exploring the criticisms of behavior therapy for tics. Clin Psychol Sci Pract. 2007;14(3):279-82.

54. Marcks BA, Woods DW, Teng EJ et al. What do those who know, know? Investigating providers' knowledge about Tourette's syndrome and its treatment. Cogn Behav Pract. 2004;11(3):298-305.

55. Shimberg EF. Living with Tourette syndrome. New York: Fireside Books; 1995.

56. Woods DW, Twohig MP, Flessner CA et al. Treatment of vocal tics in children with Tourette syndrome: investigating the efficacy of habit reversal. J Appl Behav Anal. 2003;36(1):109-12.

57. Piacentini J, Himle MB, Chang S et al. Reactivity of tic observation procedures to situation and setting. J Abnorm Child Psychol. 2006;34(5):647-56.

58. Verdellen CW, Hoogduin CA, Keijsers GP. Tic suppression in the treatment of Tourette's syndrome with exposure therapy: the rebound phenomenon reconsidered. Mov Disord. 2007;22(11): $1601-6$.

59. Burd L, Kerbeshian J. Treatment-generated problems associated with behavior modification in Tourette disorder. Dev Med Child Neurol. 1987;29(6):831-3.

60. Burd L, Kerbeshian J. Symptom substitution in Tourette disorder. Lancet. 1988;2(8619):1072.

61. Kazdin AE. Symptom substitution, generalization, and response covariation: implications for psychotherapy outcome. Psychol Bull. 1982;91(2):349-65.

62. Peterson AL, McGuire JF, Wilhelm S et al. An empirical examination of symptom substitution associated with behavior therapy for Tourette's Disorder. Behav Ther. in press.

63. Woods DW, Conelea CA, Himle MB. Behavior therapy for Tourette's disorder: utilization in a community sample and an emerging area of practice for psychologists. Prof Psychol Res Pract. 2010;41(6):518-25.

64. Scahill L, Woods DW, Himle MB et al. Current controversies on the role of behavior therapy in Tourette syndrome. Mov Disord. 2013;28(9):1179-83.

65. Flancbaum M, Rockmore L, Franklin ME. Intensive behavior therapy for tics: implications for clinical practice and overcoming barriers to treatment. J Dev Phys Disabil. 2011;23(1):61-9.

66. Blount TH, Lockhart A-LT, Garcia RV et al. Intensive outpatient comprehensive behavioral intervention for tics: a case series. World J Clin Cases. 2014;2(10):569.

67. Himle MB, Olufs E, Himle $\mathrm{J}$ et al. Behavior therapy for tics via videoconference delivery: an initial pilot test in children. Cogn Behav Pract. 2010;17(3):329-37.

68. Himle MB, Freitag M, Walther $\mathrm{M}$ et al. A randomized pilot trial comparing videoconference versus face-to-face delivery of behavior therapy for childhood tic disorders. Behav Res Ther. 2012;50(9): $565-70$. 
69. Ricketts EJ, Bauer CC, Ran D et al. Pilot open case series of voice over internet protocol-delivered assessment and behavior therapy for chronic tic disorders. Cogn Behav Pract. In press.

70. Ricketts EJ, Goetz AR, Capriotti MR et al. A randomized waitlistcontrolled pilot trial of voice over Internet protocol-delivered behavior therapy for youth with chronic tic disorders. J Telemed Telecare. In press.

71. Rowe J, Yuen HK, Dure LS. Comprehensive behavioral intervention to improve occupational performance in children with Tourette disorder. Am J Occup Ther. 2013;67(2):194-200.

72. Chang S, Himle MB, Tucker BTP et al. Initial psychometric properties of a brief parent-report instrument for assessing tic severity in children with chronic tic disorders. Child Family Behav Ther. 2009;31(3):181-91.

73. Storch EA, De Nadai AS, Lewin AB et al. Defining treatment response in pediatric tic disorders: a signal detection analysis of the Yale Global Tic Severity Scale. J Child Adolesc Psychopharmacol. 2011;21(6):621-7.

74. Jeon S, Walkup JT, Woods DW et al. Detecting a clinically meaningful change in tic severity in Tourette syndrome: a comparison of three methods. Contemp Clin Trials. 2013;36(2): 414-20.

75. Franklin ME, Best SH, Wilson MA et al. Habit reversal training and acceptance and commitment therapy for Tourette syndrome: a pilot project. J Dev Phys Disabil. 2011;23(1):49-60.

76. Lyon GJ, Samar SM, Conelea C et al. Testing tic suppression: comparing the effects of dexmethylphenidate to no medication in children and adolescents with attention-deficit/hyperactivity disorder and Tourette's disorder. J Child Adolesc Psychopharmacol. 2010;20(4):283-9.

77. McGuire JF, Lewin AB, Storch EA. Enhancing exposure therapy for anxiety disorders, obsessive-compulsive disorder and post-traumatic stress disorder. Expert Rev Neurother. 2014;14(8):893-910.

78. Storch EA, Morgan JE, Caporino NE et al. Psychosocial treatment to improve resilience and reduce impairment in youth with tics: an intervention case series of eight youth. J Cogn Psychother. 2012;26(1):57-70.

79. McGuire JF, Arnold E, Park JM et al. Living with tics: reduced impairment and improved quality of life for youth with chronic tic disorders. Psychiatry Res. 2014;225(3):571-9. 